\title{
ESTRATEGIAS PARA EL FORTALECIMIENTO DE LA ENSEÑANZA Y APRENDIZAJE DE LA MULTIPLICACIÓN EN EL GRADO TERCERO DE LA IED JULIUS SIEBER DE TUNJA
}

\author{
Strategies for strengthening the teaching and learning of multiplication in \\ the third grade of the IED Julius Sieber de Tunja
}

ROCÍO DEL PILAR DAZA HERRERA ${ }^{1}$

Recibido:11 de noviembre de 2019. Aceptado:15 de diciembre de 2019

DOI: http://dx.doi.org/10.21017/rimci.2020.v7.n14.a89

\section{Resumen}

La investigación realizada presentó como centro de interés el aprendizaje de la multiplicación, a partir de la implementación de una estrategia didáctica, aplicada a niños de grado tercero de Básica Primaria. Se tuvo en cuenta que para los niños es de vital importancia ser protagonistas de su propio conocimiento, es decir, cuando ellos se sienten involucrados en el diseño de las estrategias, se sienten motivados por el aprendizaje que viene en camino. El propósito estuvo enfocado a implementar estrategias pedagógicas mediadas por la lúdica, el juego y la cotidianidad, para contribuir en el aprendizaje de la multiplicación de los estudiantes del grado tercero de educación básica de la Institución Educativa Julius Sieber de la ciudad de Tunja. La metodología implementada estuvo enfocada en lo cualitativo, con tipo investigación acción, con el desarrollo de cuatro fases. Entre los resultados se percibe que, los niños se divirtieron con la estrategia, debido a la motivación que tuvieron con el uso del computador; los que tenían acceso a esta herramienta. Los que desarrollaron la estrategia en el cuaderno, se divirtieron, porque las situaciones problema enunciadas hicieron parte de su cotidianidad; en el mismo sentido, la reflexión docente acerca del proceso de elaboración, aplicación y reflexión de la estrategia, por parte del docente, enriquece los procesos.
\end{abstract}

Palabras clave. Estrategia didáctica; suma abreviada; producto.

\begin{abstract}
The research carried out presented as a center of interest the learning of multiplication, from the implementation of a didactic strategy, applied to children in the third grade of Basic Primary. It was taken into account that for children it is of vital importance to be protagonists of their own knowledge, that is, when they feel involved in the design of strategies, they feel motivated by the learning that is coming. The purpose was focused on implementing pedagogical strategies mediated by play, play and everyday life, to contribute to the learning of the multiplication of students of the third grade of basic education of the Julius Sieber Educational Institution in the city of Tunja. The implemented methodology was focused on the qualitative, with an action research type, with the development of four phases. Among the results, it is perceived that the children had fun with the strategy, due to the motivation they had with the use of the computer; those who had access to this tool. Those who developed the strategy in the notebook had fun, because the problem situations listed were part of their daily lives; In the same sense, the teacher's reflection on the process of development, application and reflection of the strategy, by the teacher, enriches the processes.
\end{abstract}

Keywords. Didactic strategy; abbreviated sum; product.

1 En curso Maestría en didáctica de la Matemática. Especialista en didáctica de la matemática. Licenciada en educación básica con énfasis en matemáticas humanidades y lengua castellana. Actualmente trabajo como docente en preescolar en el Hogar Infantil mentes creativas y grandes Talentos. ICBF. Correo electrónico: rocio.daza@uptc.edu.co 


\section{INTRODUCCIÓN}

L os conocimientos de la disciplina que el docente orienta en una Institución Educativa y los procesos didácticos que utiliza para que tales conocimientos sean asimilados por los estudiantes, es, tal vez, la clave del éxito en el proceso de enseñanza y aprendizaje. El caso que ocupa la presente investigación es, el producto, como una operación dada entre diversas cantidades, llámese números, expresiones, elementos, montos, etc. El propósito se estableció en el diseño de una estrategia didáctica, estructurada en el juego, la lúdica, el contexto, para analizar la manera cómo contribuye en el aprendizaje de la multiplicación de los estudiantes del grado tercero de educación básica.

El proceso de aprendizaje de la multiplicación en los niños, trae consigo muchas dificultades, aprender las tablas de multiplicar, el proceso del algoritmo; pero sobretodo, la manera como los estudiantes aplica el aprendizaje de las tablas y, la combinación del nuevo algoritmo con los que ya aprendió, en la solución de problemas de aplicación. Es decir, no basta con solo aprender las tablas y saber multiplicar cantidades, se debe tener conciencia de para qué le sirven esos dos elementos de la matemática. Y ese es definitivamente el problema, los niños que cursan los grados terceros, cuartos y de ahí hacia adelante, no saben interpretar, comprender, analizar, lo que dice la situación, menos traducir la respuesta que tal problema está solicitando. Algunos argumentan que los niños no saben leer, que no se les facilita la matemática, que no se aprenden las tablas, en fin; son muchas las excusas, pero en últimas los niños terminan indagando a su docente por lo que hay que hacer o por la operación que deben realizar para solucionar el asunto [1].

Se hace necesario replantear las estrategias que se han trabajado para que los niños se aprendan las tablas, música, granos de algunas legumbres, trucos con los dedos, en fin, hay muchas, se aplican, pero el problema continúa. Problemas sencillos de solucionar y a los niños les cuesta trabajo; las tablas las rezan hacia adelante hacia atrás, de memoria, pero al ir a solucionar una situación problema, no lo logran. Entonces ¿qué hacer?, es la pregunta que los docentes de básica, media y aún del ciclo universitario, se cuestionan. Los niños que desempeñan papel protagónico en el super- mercado, en la tienda, en los negocios de los padres, han logrado superar tal dificultad, hacen cuentas rápido, efectivas y sin problema. Se presume que cuando el niño sabe para qué le sirve el conocimiento que adquiere en el salón, lo asimila, lo hace propio y lo utiliza en su cotidianidad y para su beneficio [2].

Esto y muchas otras situaciones son las que han conducido a querer profundizar sobre el tema de la multiplicación, es un interés general de los docentes de básica, implementar estrategias que conduzcan al éxito en el proceso de aplicación de la multiplicación en la solución de problemas. Esta temática se ha venido convirtiendo en un escollo al que no se le ha dado solución, los resultados en las pruebas SABER, en los pruebas internas y externas, muestran un origen, se adolece de procesos que conduzcan a los niños en la aplicación de las tablas en la solución de problemas [3][4]. No se diga nada frente a la dificultad de los padres de familia, cuando del aprendizaje de las tablas se trata, hasta maltrato físico reciben los niños por no aprender las tablas. En fin son muchas las consideraciones que impulsan a tomar esta temática como centro de estudio para la presente investigación.

El trabajo se divide en seis capítulos; el primero hace énfasis en el problema de investigación, allí se exponen algunas problemáticas que se han detectado en torno al tema mencionado y que son analizadas desde la experticia de la docente investigadora, se plantea la pregunta que orientó la investigación, los objetivos y cierra con la justificación, es decir, el por qué se adelantó este proceso. En el segundo capítulo se trabajó lo concerniente al marco de referencia, allí se expusieron los trabajos previos a esta investigación y que han trato de solucionar la problemática, se retomaron trabajos en el orden internacional, nacional y local. El análisis condujo a establecer tres categorías, a saber, la multiplicación, las tablas de multiplicar y los medios tecnológicos, con ocasión de la pandemia por la que atraviesa la humanidad. En el marco teórico, por tratarse de una maestría en didáctica, se hace especial énfasis en los diversos procesos didácticos de la educación matemática y en la estrategia didáctica como herramienta de trabajo.

El tercer capítulo muestra o da a conocer lo concerniente a la metodología de investigación, allí se expone el enfoque, el tipo de investigación, las fases, 
la línea de investigación; en el cuarto se realiza lo correspondiente al análisis de la información recabada; para esta parte del trabajo se hizo uso del software SPSS. En el quinto capítulo se establece la discusión de los resultados que fueron coherentes con los instrumentos aplicados. El trabajo finaliza con las conclusiones, las referencias y los anexos.

\section{Marco teórico/CONCEPTUAL}

Este proyecto nace de la inquietud por cambiar actitudes tanto de estudiantes como de docentes por los problemas expuestos, dentro del proceso de enseñanza y aprendizaje de una de las asignaturas fundamentales como son las matemáticas, específicamente en el tema de la multiplicación.

La necesidad del estudio se basa en la problemática que se observó entre los niños del grado tercero de la institución educativa en mención, y en investigaciones de la educación infantil en las que se evidencia como un mal aprendizaje de la multiplicación puede obstaculizar otros procedimientos matemáticos, como por ejemplo la operatividad en la solución de problemas [5].

En este espacio del marco, se tratará lo concerniente a los aspectos teóricos que soportan la investigación; entre otros aspectos didácticos como la teoría de los campos conceptuales de Gérard Vergnaud y su correspondencia en la enseñanza y la investigación de las matemáticas; de igual manera se abordó lo concerniente a la estrategia didáctica [6]. Su importancia radica en que se plantea una solución a la problemática, mediante la enseñanza de la multiplicación a través de intervenciones pedagógicas como la lúdica, el juego y la cotidianidad, que lleven a un aprendizaje significativo de esta operación matemática. Para lograr el objetivo se aplicó una conducta de entrada, conducta de salida y unas secuencias didácticas.

La teoría de los campos conceptuales en la matemática ha sido elaborada principalmente para dar cuenta a procesos de conceptualización de las estructuras aditivas, multiplicativas, relación numérica, espacio y el álgebra; también se realiza un proceso donde se relaciona el concepto y el esquema la cual ayuda en la resolución de problemas que es elaborada para trabajarla parte psicológica y la didáctica teórica - práctica [7].
Los campos conceptuales son referentes para la enseñanza de la ciencia y la investigación e áreas donde se hablan de aprendizajes significativos, resolución de problemas y representaciones mentales que son trabajados por dos autores muy importantes que trabajan por aspectos importantes sobre las matemáticas involucrando las operaciones básicas como la adición, multiplicación, división, funciones lineales, funciones no lineales, espacios vectoriales, análisis dimensional, fracción, razón, tasa, números racionales, donde el dominio requiere una adición, una sustracción o una combinación de las operaciones [8].

La matemática como la ciencia van de la mano y sus campos conceptuales que se trabajan ayudan a superar las dificultades presentadas al ejecutar diversas problemáticas logrando dar solución a dichas inquietudes que le permiten al estudiante lograr identificar el problema y experimentar diversas soluciones dando como finalidad una resolución lógica - matemática involucrando conceptos, operaciones, análisis, campo y error para lograr lo que se requiere solucionar [9]. Otros aportes de Piaget, Vergnaud, Vigosky podemos plantear soluciones matemáticas aportando conceptos, operaciones, efectuando análisis, descartando probabilidades, dando solución a lo que nos requieren en las problemáticas presentadas a nivel matemático ayudando al estudiante de una forma sencilla, clara y fácil de analizar para llegar a su fin o conclusión [10].

La estrategia didáctica, es definida "como un proceso integral que organiza y desarrolla un conjunto de acciones que se proyectan y se ponen en marcha de forma ordenada para alcanzar un determinado propósito pedagógico"[10]. Las estrategias didácticas según componente cognitivo involucran actividades que propicien el desarrollo de habilidades cognitivas y la construcción del conocimiento matemático; las estrategias didácticas según componente afectivo promueven el desarrollo afectivo de los estudiantes en relación con sus creencias, actitudes y emociones, las cuales, a su vez, están vinculadas con el aprendizaje de la Matemática [11].

El desarrollo de este apartado permite un acercamiento a la conceptualización de estrategia didáctica, como herramienta que permite fomentar los procesos de enseñanza y aprendizaje adelanta- 
dos por estudiantes y docentes. De esta manera, la importancia de la didáctica en los procesos de enseñanza-aprendizaje, y educativos de todo nivel, es crucial; y es que cuando se habla de didáctica, se habla de la "disciplina de la pedagogía que estudia y perfecciona los métodos, procesos, técnicas y estrategias cuyo objetivo es potenciar la enseñanza para lograr aprendizajes más amplios, profundos y significativos" [12].

\section{DiseÑo METODOLÓGICO}

El presente estudio se desarrolló en el marco de un enfoque Cualitativo, con este enfoque, se aplicó una estrategia didáctica en la que se observaron los resultados obtenidos en el proceso, dando así respuesta a la pregunta de investigación y planteando logros sobre los objetivos propuestos. Se trató de una investigación cualitativa indagatoria porque se mueve de manera dinámica en dos sentidos; entre los hechos y su interpretación [13]. En la línea del enfoque cualitativo, se encuentra la Investigación Acción, el proceso educativo se convierte en una práctica investigativa, donde el docente es el principal investigador de su propia práctica; la indagación, su formación continua con miras a fortalecer la calidad de la educación, las dificultades en el aula, la reflexión de sus prácticas, tienen un impacto en el aprendizaje significativo de los estudiantes.

Para la investigación se describen cuatro fases:

Primera fase: determina los problemas de aprendizaje de la multiplicación de los estudiantes del grado tercero, mediante una prueba diagnóstica, consta de preguntas cerradas y problemas tipo saber. Link prueba diagnóstica:

https:/ / docs.google.com/forms/d/1bgHDy LuhqcrHwlT2eE0cmPfmXSRsSCWT4vyt5Q5xD $\mathrm{Sc} /$ prefill

Segunda fase: se diseñó y aplicó una secuencia didáctica "Multiplicando" conformada por talleres, con actividad lúdicas y una guía didáctica para la docente a través de videos. Link secuencia didáctica:

https://docs.google.com/forms/d/1vTzmjYd qLOQOyNZ-9Xwg3iQo9PgRwvoqZIJV8Bit ObI/edit
Link videos:

La multiplicación:

https://www.powtoon.com/s/dqM5O38Mp $\mathrm{I} 0 / 1 / \mathrm{m}$

Propiedades de la multiplicación;

https://www.powtoon.com/s /f7TGWnIM $\mathrm{DP} 9 / 1 / \mathrm{m}$

Método Polya:

https://www.powtoon.com/s/bLXKyN4 $\mathrm{mhlk} / 1 / \mathrm{m}$

Tercera fase: la fase de observación, hace parte de la anterior fase, se observó la incidencia de la estrategia didáctica, mediante una comparación de la conducta de entrada y la prueba tipo saber (Anexo 1 y Anexo 2)) y la de salida (Anexo 3), detectando así tanto los problemas iniciales y el avance con lo pedagógico. Link: caracterización de estudiantes y prueba docente.

https://docs.google.com/forms/d/1wYn525 r7eO_YxezdhU3iSftThKDMizDrknuRGueSQ $\mathrm{Lg} /$ edit

https:/ / docs.google.com/forms/d/10KbQx glbFqN4vjRo7qBDws4Bm9uC2G10epKr9EeVU Wk/edit?usp=drive_web

Cuarta fase: la fase de reflexión se puede observar en los análisis de los instrumentos aplicados y en la estructura del informe final, basados tanto en los resultados de las secuencias como en las actitudes de los estudiantes.

\section{Análisis de la Información}

Para el análisis de la información de las diversas actividades aplicadas en cada una de las fases, se hizo uso del programa estadístico SPSS, que se trata de un software que es utilizado para realizar la captura y análisis de datos para crear tablas y gráficas con data compleja. ElSPSS es conocido por su capacidad de gestionar grandes volúmenes de datos y es capaz de llevar a cabo análisis de texto entre otros formatos más. 


\section{Análisis de Resultados}

\section{Encuesta docente}

El análisis se realizó tomando cada una de las preguntas, haciendo el respectivo gráfico, A la pregunta 1. ¿Cuál es su formación profesional? La mitad de los docentes manifestaron ser licenciados, una tercera parte poseen estudios de especialización y una sexta parte manifestó ser magister. La Fig. 1 muestra la distribución de los docentes entrevistados en un diagrama de barras.

En esta respuesta, se puede resaltar que, a mayor nivel de formación, poseen variadas herramientas didácticas para compartir el conocimiento. En el caso que ocupa la investigación, los docentes que ostentan estudios de posgrado, gozan de más posibilidades para motivar, promover, infundir el deseo de aprender en sus estudiantes.

A la pregunta ¿Durante cuántos años ha ejercido la docencia?, de los docentes entrevistados, la mitad cumple con cinco años de experiencia, la otra mitad, es decir tres de ellos oscilan con una experiencia entre los 6 y los 16 años.

La entrevista se realizó mediante el uso de formularios google, estuvo conformada por quince preguntas, que indagan desde la formación, la experticia docente, hasta los aspectos fundamentales que son tenidos en cuenta por ellos al momento de socializar el algoritmo de la multiplicación; se aplicó a seis docentes de la institución Educativa Julios Sieber de la ciudad de Tunja.

El análisis arroja que, cada docente tiene una manera de pensar al respecto; esto podría significar, al igual que el punto anterior, que cada docente planea con rumbos diferentes, que no existe una verdadera comunidad de aprendizaje, donde planean, aplican, reflexionan, evalúan, entre otras.

Se hace necesario que los docentes caminen en una misma dirección, en el currículo, en las estrategas de clase, en el diseño de estrategias, en la evaluación; solo de esa manera, los niños mos-

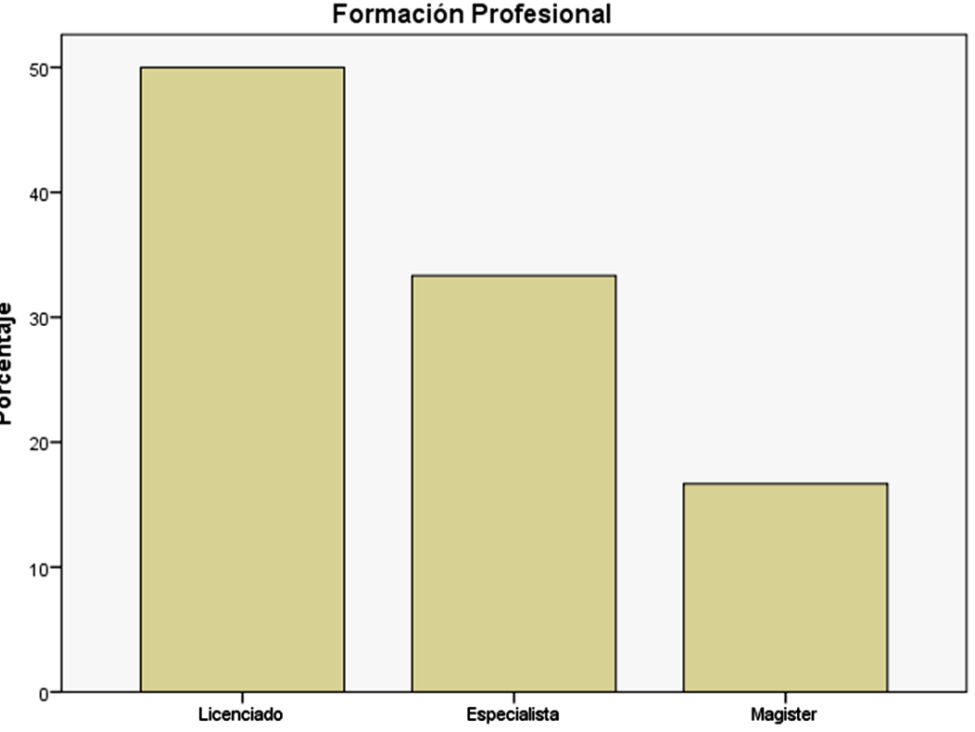

Fig. 1. Formación profesional de los docentes entrevistados

trarán avances significativos en los procesos de aprendizaje.

\section{Análisis de la encuesta de caracterización a estudiantes}

La primera pregunta indaga a los estudiantes acerca de cinco cosas buenas de aprender matemáticas; los estudiantes consideran que, saber operar cantidades, desarrollar la inteligencia, desempeñarse en la vida cotidiana, son entre otros, los aspectos afables de tener conocimientos matemáticos. En un segundo plano, favorecen el conocimiento de cantidades, para jugar, para desarrollar la inteligencia; un estudiante manifiesta que no le sirve para nada. Fig. 2.

La encuesta para caracterizar a los estudiantes se realizó mediante el uso de formularios google; se aplicó a 32 estudiantes de la Institución Educativa Julios Sieber de la ciudad de Tunja; estuvo conformada por once preguntas abiertas que indagan aspectos generales del producto, la utilización que los niños le otorgan, cómo la asimilan, en que aspectos de la cotidianidad les es más útil, por qué se les dificulta, entre otros aspectos.

Como conclusión, los estudiantes consideran que, saber operar cantidades, desarrollar la inteligencia, desempeñarse en la vida cotidiana, son entre otros, los aspectos afables de tener conocimientos mate- 
máticos. En un segundo plano, favorecen el conocimiento de cantidades, para jugar y para desarrollar la inteligencia.

\section{Análisis de la prueba diagnóstica}

La pregunta uno, Explique con sus propias palabras ¿qué es una multiplicación?. Los estudiantes manifiestan que es una operación, una suma abreviada, una respuesta, en fin. En la figura 3 se puede observar la forma como dieron respuesta a esta inquietud.

Se observa, que, para la mayoría de los niños, la multiplicación se trata de una operación; algunos la consideran como sumar varias veces el mismo número y otros, es la que permite obtener un resultado. En el trasfondo de las respuestas, no se obtiene mayor ilustración acerca de lo que realmente es la multiplicación.

La siguiente situación: Dada la operación $5 \times 3=15$. Escriba cual es el producto, escriba cuáles son los factores. El alto número de niños contesta acertadamente las dos preguntas, ver Fig. 4.

La mayoría de los niños responde acertadamente, tanto al producto, como a sus factores; sin embargo, a los que presentan dudas, se les debe hacer un refuerzo en lo concerniente a los términos de la multiplicación. Uno de tantos problemas en la multiplicación es precisamente ese, los niños confunden las definiciones de producto y de factores; además, se hace necesario

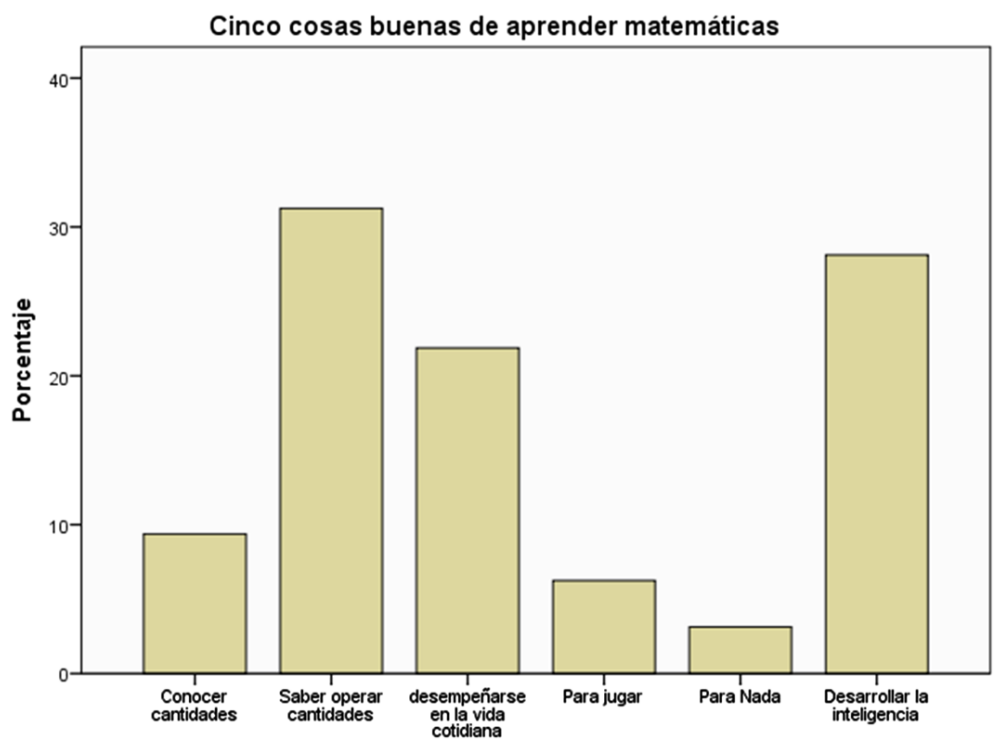

Fig. 2. Cosas buenas de aprender matemáticas

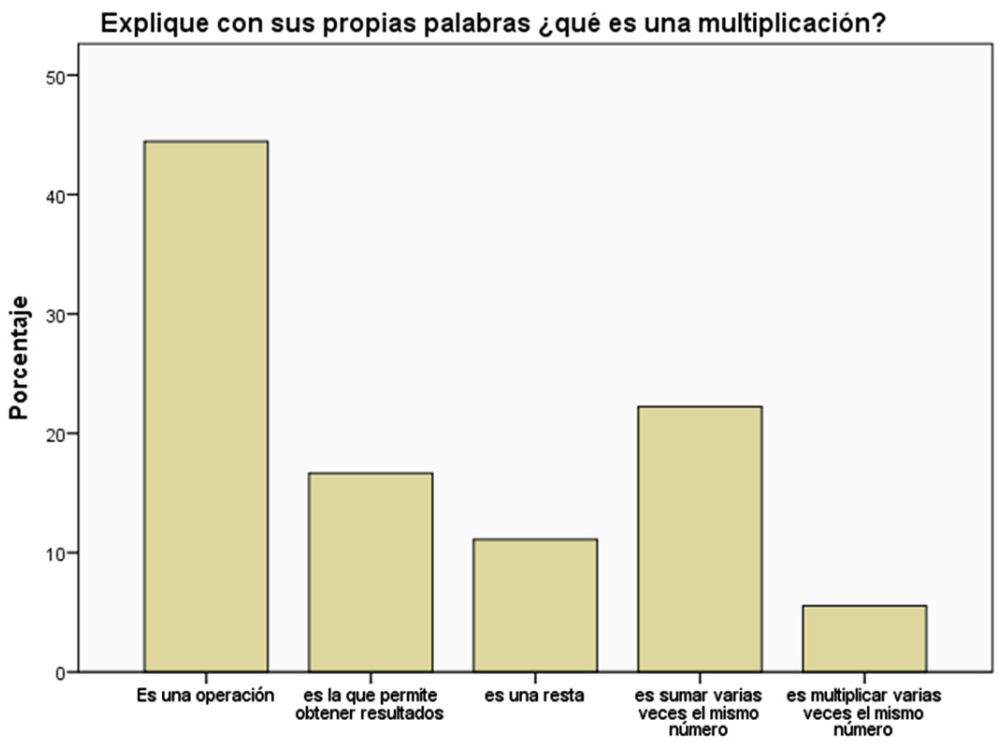

Fig. 3. Explique con sus propias palabras ¿qué es una multiplicación? que ellos comprendan la importancia que reviste cada uno de los factores en la operación de la multiplicación y, sobre todo, puede llegar a afectar la situación planteada.

En la siguiente situación: $\mathrm{Si} 3+3+3+3+3$ es equivalente a 5 veces 3 , es decir $5 \times 3$. Explique cómo se puede expresar $4+4+4+4$ y cuál es el resultado. Los estudiantes mostraron las siguientes respuestas: $4 \times 4$ $=16 ; 4$ elevado a 4 es igual $16 ; 4$ veces $4=16$, es decir $4 \times 4=16$; se nota que gran parte de los niños tienen claridad, excepto en la manifestación de 4 elevado a 4 es igual 16, se evidencia una confusión entre el significado de producto y el de potencia.

Este diagnóstico se aplicó vía google, 18 estudiantes de la Institución Educativa Julios Sieber lo respondieron; consta de 18 preguntas, donde se indaga acerca de la conceptualización y algunas aplicaciones que los estudiantes tienen del concepto de la multiplicación. Se analiza mediante el uso del SPSS en las preguntas que lo permiten y por observación y cotejo en las que así lo requieren.

Ahora bien, con base en las respuestas de los estudiantes y teniendo en cuenta que se trata de problemas aritméticos que presentan datos en forma de cantidades y, establecen entre ellos relaciones de tipo cuantitativo, donde solo necesitan la 

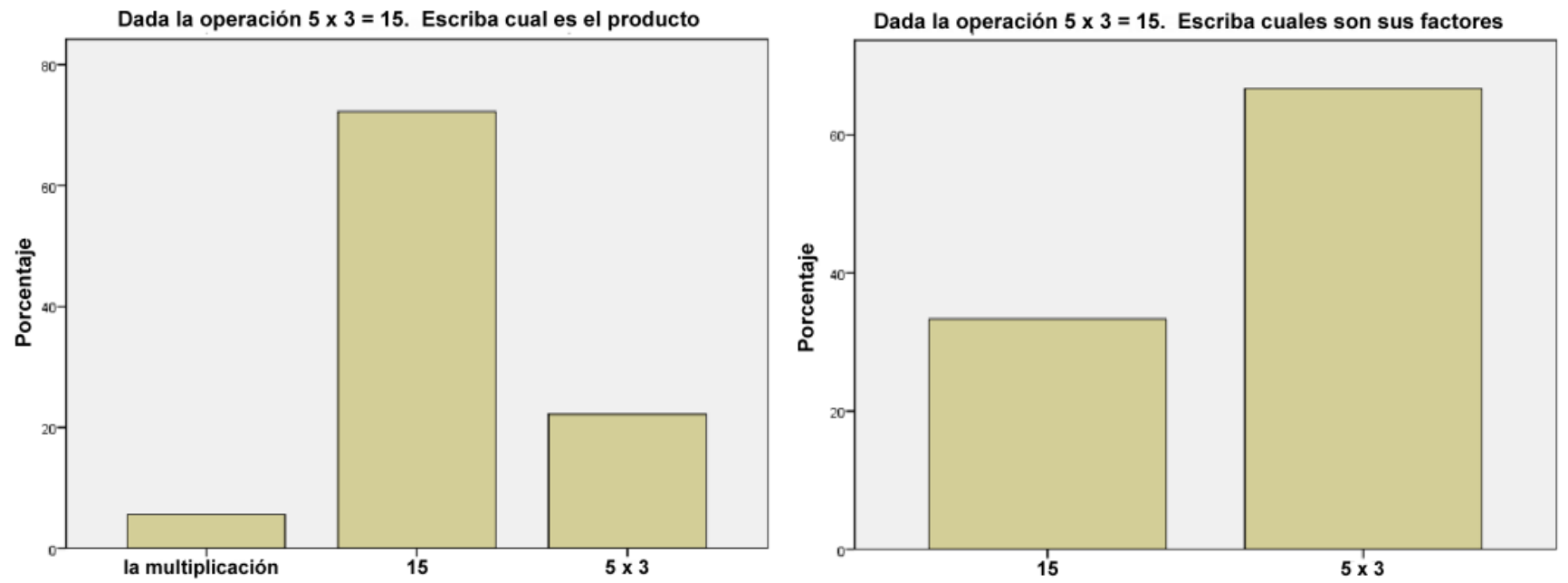

Fig. 4. Dada la operación 5x3=15, ¿Cuál es el producto? ¿Cuáles son los factores?

realización de operaciones aritméticas para encontrar la solución, en este caso, solo necesitaban realizar una multiplicación; son pocos los niños que alcanzan una respuesta razonable a la inquietud del problema. Lo anterior conduce a pensar que el proceso de resolución de problemas debe ser uno de los ejes principales del aprendizaje matemático, puesto que en estos procesos los alumnos utilizan diferentes capacidades básicas tales como leer de manera comprensiva, reflexionar, establecer un plan de trabajo, modificarlo si fuese necesario y comprobar la o las soluciones.

\section{Análisis de la secuencia didáctica}

En la primera situación, se plantea que: en la clase de dibujo, Juan y Sara hicieron caritas de perro con papel y las empacaron en cajas como lo ilustra la Fig. 5.

Para saber el total de caritas de papel que Sara y Juan realizaron, se propone como alternativa para resolver esta operación. El 84.5\% de los niños contesto que Multiplicar $4 \times 6$, porque 4 grupos de 6 unidades conforman 24 unidades; un $7.7 \%$ manifestaron que debían multiplicar $6 \times 4$, porque hay 6 cajas con 4 caritas de papel cada una, respuesta errada y, el otro $7.7 \%$ afirma que deben multiplicar el número total de cajas por la cantidad de caritas de papel de perro, ver Fig. 6.

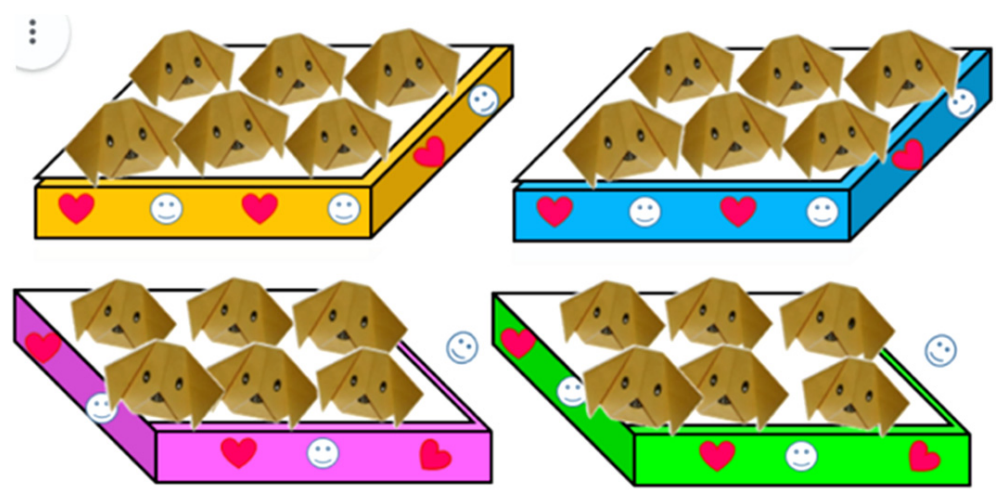

Fig.5. Situación problema uno de la estrategia didáctica

Para saber el total de caritas de papel que Sara y Juan realizaron, se propone como alternativa para resolver esta operación.

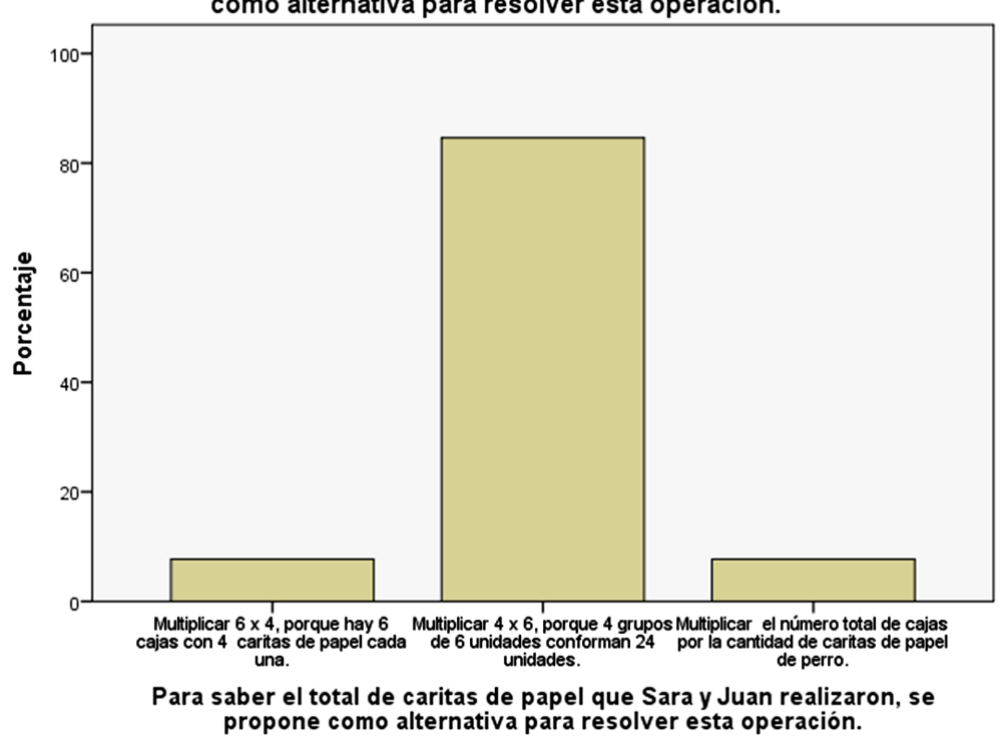

Fig. 6. Diagrama de barras de la situación uno de la estrategia didáctica 
Se trata de un problema de primer nivel con una estructura multiplicativa, donde se evidencia un factor " $\mathrm{N}$ " o comparación multiplicativa, el problema expresa la regla de proporción entre las caras de perro y las cajas donde las empacaron, dada la cantidad de caras de perro (multiplicando) y la cantidad de cajas (multiplicador), se pregunta por la cantidad resultante (producto) de la misma naturaleza que el multiplicando.

Las situaciones quinta y sexta se debían solucionar a partir de la siguiente información: Si un número se puede representar por más de un rectángulo el número es compuesto. El número 8 es un número compuesto y se puede representar mediante un rectángulo de lado 4 y altura 2 , o un rectángulo de lado 8 y altura 1 , como muestra la Fig. 7.

La situación cinco solicita construir rectángulos que representen el número 36, capture la imagen y adjúntela como archivo en este sitio. Fig. 8.

Los arreglos presentados por todos los estudiantes cumplen con el propósito del ejercicio, se puede inferir que se trata de una alternativa utilizada para el aprendizaje de las tablas de multiplicar. Un arreglo rectangular, como su nombre lo dice, es un rectángulo que contiene rectángulos más pequeños en su interior, dispuestos de manera perfecta, es decir, no sobran ni faltan rectángulos pequeños. En realidad, siempre que se cuenten elementos que están dispuestos como arreglo rectangular, basta con contar el número de filas y el número de columnas y luego multiplicar.

En la sexta situación, solicita a los niños que, elija la lista en la cual aparezcan números que no puedan ser representados por más de un rectángulo. La manera como los estudiantes emitieron sus respuestas están en la figura 9.

Se evidencia que todas las posibilidades de respuesta son correctas; sin embargo, en la opción $\{4,7,9,17,100\}$ se muestran los números 7 y 17 que son primos y presentan una única posibilidad de
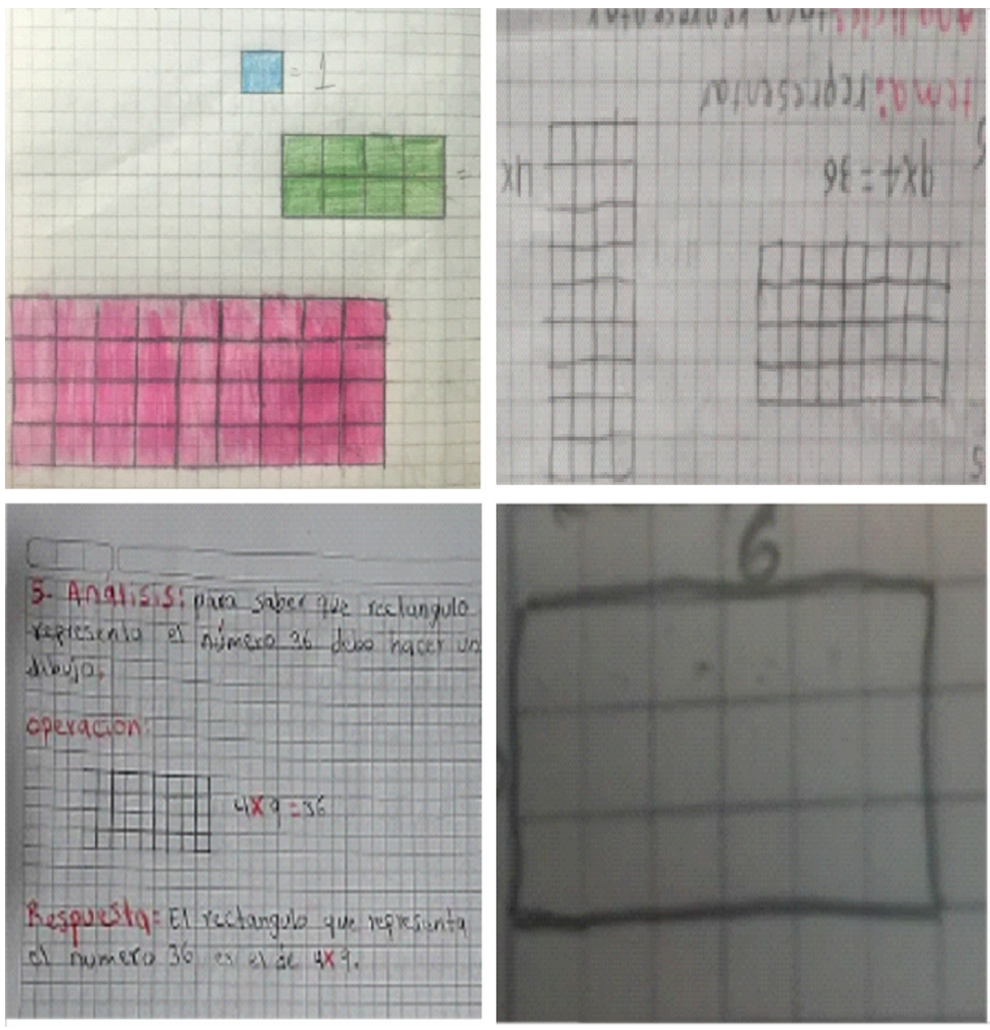

Fig. 8. Arreglos de números compuestos realizados por los estudiantes.

arreglo. Las demás opciones de respuesta tienen más de un arreglo rectangular, incluyendo donde aparece el número 9, el tiene dos posibilidades, $9 \times 1$ y $3 \times 3$. Solo el $30.8 \%$ de los estudiantes tomaron la respuesta $\{4,7,9,17,100\}$.

La Secuencia Didáctica "Multiplicando" se realizó mediante el uso de formularios google; se aplicó a 13 estudiantes de la Institución Educativa Julios Sieber de la ciudad de Tunja; estuvo conformada por 19 situaciones aditivas y multiplicativas en diversos contextos. 


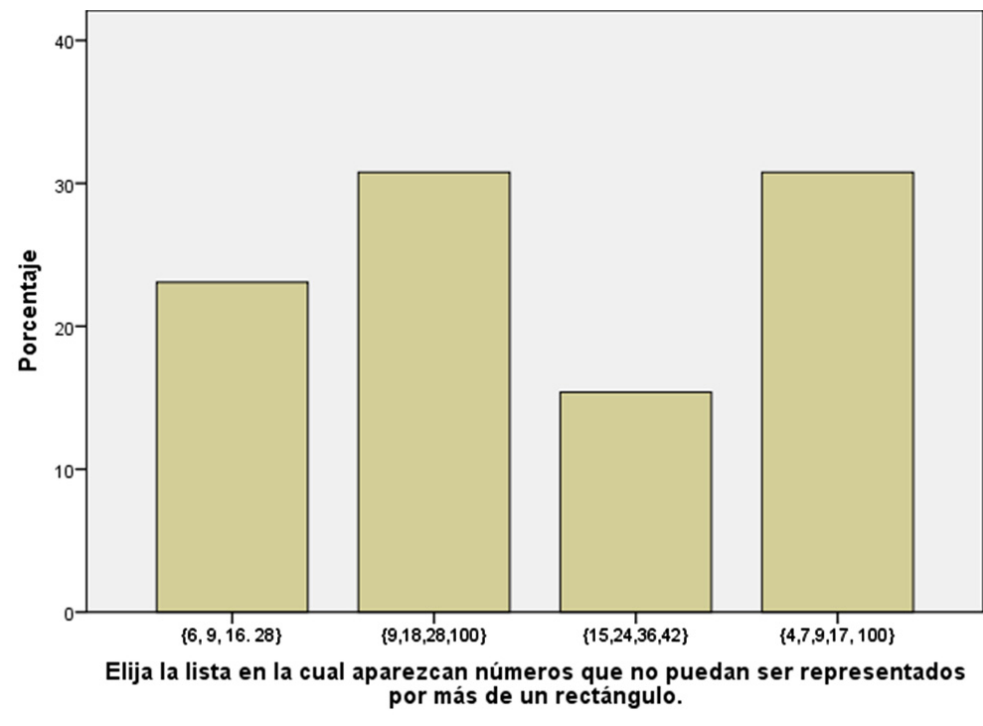

Fig. 9. Diagrama de barras para la sexta situación de la estrategia didáctica. cotidianidad, contribuyen en el aprendizaje de la multiplicación de los estudiantes del grado tercero de educación básica de la Institución Educativa Julius Sieber de la ciudad de Tunja. Frente a este propósito se pudo establecer que, los niños se divirtieron con la estrategia, debido a la motivación que tuvieron con el uso del computador; los que tenían acceso a esta herramienta. Los que desarrollaron la estrategia en el cuaderno, se divirtieron, porque las situaciones problema enunciadas hicieron parte de su cotidianidad.

Es la experiencia que queda luego de aplicar este proceso, cuando el maestro diseña estrategias con situaciones propias de la cotidianidad de los niños, ellos se identifican tales estrategias, las compren-

El propósito de la estrategia didáctica fue caracterizar situaciones aditivas y multiplicativas en diversos contextos y representaciones de los componentes numérico variacional y espacial métrico vinculados a las competencias de comunicación, razonamiento y resolución de problemas.

Se observó el desarrollo de la actividad con la obtención de mejores resultados en el proceso de solución; los estudiantes tenían mayores conocimientos del proceso de solucionar un problema de contexto, mediante la aplicación de dos o tres productos de manera simultánea; los estudiantes estuvieron motivados en el proceso, mostraron agradecimiento y felicidad por las actividades diseñadas; mientras que, la docente titular dio muestras de agrado por el diseño de las actividades

Se pudo concluir que el conocimiento acertado del contenido es de vital importancia a la hora de abordar las temáticas con los estudiantes; no se puede dejar de lado el horizonte didáctico, este brinda las herramientas para mantener a los estudiantes, interesados, motivados, despiertos, en la ejecución de las actividades; de esta manera se alcanzan mejores resultados en los procesos educativos.

\section{CONCLUSIONES}

El objetivo general propone implementar una estrategia mediada por la lúdica, el juego y la den, las hacen suyas, no se sienten presionados, los gusta, están motivados y logran un aprendizaje significativo [14]. Se hace necesario que el docente conozca a fondo el contexto de sus estudiantes, de esa manera podrá diseñar estrategias concernientes a la realidad del alumno, para que, de esa manera, el niño se sienta identificado con el conocimiento que adquiere en el aula de clase.

$\mathrm{Al}$ primer objetivo específico, caracterizar los conocimientos sobre multiplicación, en la población objeto de estudio; se pudo determinar que los niños tienen dificultades en el proceso lector, ellos deletrean, pero no leen, por tal razón, no comprenden lo que leen, menos serán conscientes de analizar y saber qué es lo que el problema o la situación les esta indagando. El trabajo con uso de plataformas, es llamativo para ellos, por el mismo uso del computador, se interesan por presentar las actividades.

Se diseñó y aplicó la estrategia a partir del algoritmo del producto con los estudiantes de la Institución Educativa, en ella se plantearon problemas del contexto de los estudiantes, situación que los mantuvo interesados en los diversos procesos que allí se solicitaron. Esto lleva a concluir que el estudiante se siente protagonista de su propio conocimiento cuando, en las herramientas diseñadas para tal fin, él es participe, su contorno es mencionado, se trató de situaciones que lo involucran, como un actor más de la comunidad. 
La valoración de la estrategia, la evaluación de la contribución de las estrategias implementadas para el fortalecimiento de la convivencia en el colegio, es el tercer objetivo específico. Se concluye que la estrategia arroja buenos resultados, en el proceso de solución de las problemáticas expuestas, los niños acertaron y demostraron la manera efectiva de encontrar la solución; es decir, mostraron los procesos empleados para alcanzar la respuesta acertada, eso es muestra de que lograron entender adecuadamente la temática. Se fortalece la convivencia por el uso de las herramientas tecnológicas, el computador, el Whatsapp, los videos que hicieron para evidenciar sus trabajos, son situaciones que hacen que ellos compartan al interior de sus familias y con sus compañeros.

Por último, se realizó una guía didáctica a la docente explicando conceptos sobre la multiplicación, sus factores y propiedades. También se realizó con base en el método Polya un paso a paso de un ejemplo para la resolución de problemas.

\section{REFERENCIAS}

[1] J. Quintana, Trabajo de grado. El uso de las TICs para mejorar las operaciones básicas de números enteros en segundo grado de educación primaria. Tarapoto, Universidad Nacional de San Martín, Perú. 2018.

[2] M. Rodríguez y J. González, Las tablas de multiplicar con sabor a juego. Recurso didáctico. Números, V. 30. pp. 7-19. 2015.

L. Botero, E. Andrade y L. Andrade, La crisis de la multiplicación: Una propuesta para la estructuración conceptualVoces y Silencios: Revista Latinoamericana de Educación, Vol. 2, No. especial, 38-64 ISSN: 2215-8421. 2011.

[3] MEN. Estándares básicos de competencias matemáticas. Bogotá: MEN. 2006.
[4] ICFES. Guía de Orientación. Obtenido de: https:// www.icfes.gov.co/documents/20143/1353827/ Guia+de+orientacion+saber+3+2017.pdf/ 88661c7d-f31a-b31c-afca-8e38532d7e4f. 2017.

[5] A. Espeleta y A. Fonseca, Estrategias didácticas: un componente de la planificación de la lección de matemáticas. IX Festival internacional de matemáticas, (págs. 1-19). Quepos-Costa Rica. 2014.

[6] C. Fernández, Principales dificultades en el aprendizaje de las Matemáticas. Pautas para maestros de educación primaria. Chile: Universidad Internacional de La Rioja. 2013.

[7] R. Figueroa, C. Utria y R. Colpas, Estudio exploratorio de las interacciones mentales de los estudiantes de sexto grado sobre el entendimiento del concepto multiplicación. Educación y pedagogía, Vol. 17., N. 43., pp. 109-125.1997.

[8] A. García, La maestría: una opción de formación docente para Educación Media Superior y Superior en México . Praxis Educativa, vol. 19, núm. 2, pp. 19-26. 2015.

[9] A. Grisales, Uso de recursos TIC en la enseñanza de las matemáticas: retos y perspectivas . Educación, vol.14, No 2, p.198-214. 2018.

[10] S. Salazar, El conocimiento pedagógico del contenido como modelo de mediación docente. Costa Rica: CECC/SICA. 2012.

[11] L. Gutiérrez, Los ambientes de aprendizaje en la clase: dispositivo fundamental para favorecer las competencias matemáticas en niños de educación primaria . Educando para educar , 18., ejemplar 33, pp. 29-41. 2017.

[12] C. Muñoz, Trabajo de Especialización. Estrategias didácticas para desarrollar el aprendizaje significativo de las tablas de multiplicar en niños del grado tercero de EBP. . Chía, Universidad de la Sabana, Colombia. 2010.

[13] R. Hernandez, C. Fernandez, y M. Baptista, Metodología de la Investigación (Sexta ed.). México: McGRAW-HILL / Interamericana Editores. 2014.

[14] J. Godino, C. Batanero, Fundamentos de la enseñanza y aprendizaje de las matemáticas para maestros. Granada : Universidad de Granada. 2003. 